\title{
Eficácia e segurança do dupilumabe no tratamento da rinossinusite crônica com polipose nasal
}

\author{
Efficacy and safety of dupilumab in the treatment of chronic rhinosinusitis with nasal polyposis
}

Juliana F. Bianchini Garcia ${ }^{1}$, Pedro Giavina-Bianchi ${ }^{1}$

\section{RESUMO}

A rinossinusite crônica (RSC) é uma síndrome caracterizada pela inflamação da mucosa nasal e dos seios paranasais por pelo menos 12 semanas, acometendo de $5 \%$ a $12 \%$ da população geral. A síndrome é associada a alta morbidade e considerada um grande problema de saúde pública devido a sua prevalência, seu custo para a sociedade e ao impacto que acarreta na qualidade de vida dos pacientes e em seu desempenho escolar ou profissional. Ademais, a RSC está associada a diversas comorbidades, como dermatite atópica, distúrbios respiratórios do sono, conjuntivite, otite média, asma e problemas emocionais. O dupilumabe é eficaz e seguro no tratamento da RSC com polipose nasal. A eficácia é progressiva no primeiro ano de tratamento, e a posologia de $300 \mathrm{mg}$ a cada duas semanas é superior em relação à de cada quatro semanas. A interrupção do tratamento com 24 semanas acarreta a perda parcial de seus efeitos benéficos. $O$ imunobiológico também é eficaz no controle da asma nos pacientes que apresentam essa doença como comorbidade. Alguns pacientes podem apesentar aumento transitório de eosinófilos sanguíneos, e $2,7 \%$ desenvolveram conjuntivite como reação adversa nos estudos SINUS-24 e SINUS-52. O dupilumabe é uma excelente opção terapêutica no tratamento concomitante de múltiplas doenças caracterizadas pela inflamação de tipo II.

Descritores: Rinite, sinusite, pólipos nasais, células Th2, anticorpos monoclonais.

A rinossinusite crônica (RSC) é uma síndrome caracterizada pela inflamação da mucosa nasal e dos seios paranasais por pelo menos 12 semanas, acometendo 5-12\% da população geral. A síndrome é associada a alta morbidade e considerada um grande problema de saúde pública, devido a sua prevalência,

\section{ABSTRACT}

Chronic rhinosinusitis (CRS) is a syndrome characterized by inflammation of the nasal mucosa and paranasal sinuses for at least 12 weeks, affecting $5 \%$ to $12 \%$ of the general population. The syndrome is associated with high morbidity and is considered a major public health problem because of its prevalence, its cost to society, and the impact it has on patients' quality of life and on their school or professional performance. Furthermore, CRS is associated with several comorbidities, such as atopic dermatitis, sleep-disordered breathing, conjunctivitis, otitis media, asthma, and emotional problems. Dupilumab is effective and safe in the treatment of CRS with nasal polyposis. Effectiveness is progressive in the first year of treatment, and a dosage of $300 \mathrm{mg}$ every two weeks is more effective than that of every four weeks. Discontinuing treatment at 24 weeks results in partial loss of its beneficial effects. The biological drug is also effective in controlling asthma in patients who have this disease as a comorbidity. Some patients may experience a transient increase in blood eosinophils, and $2.7 \%$ developed conjunctivitis as an adverse reaction in the SINUS-24 and SINUS-52 studies. Dupilumab is an excellent therapeutic option in the concomitant treatment of multiple diseases characterized by type II inflammation.

Keywords: Rhinitis, sinusitis, nasal polyps, Th2 cells, monoclonal antibodies.

seu custo para a sociedade e impacto que acarreta na qualidade de vida dos pacientes e em seu desempenho escolar ou profissional. Ademais, a RSC está associada a diversas comorbidades, como dermatite atópica, distúrbios respiratórios do sono, conjuntivite, otite média, asma e problemas emocionais ${ }^{1}$.

1. Disciplina de Imunologia Clínica e Alergia, Universidade de São Paulo - São Paulo, SP, Brasil.

Submetido em: 10/02/2021, aceito em: 26/02/2021.

Arq Asma Alerg Imunol. 2021;5(3):232-6.

http://dx.doi.org/10.5935/2526-5393.20210038 
O diagnóstico da RSC é baseado em critérios clínicos e laboratoriais (Tabela 1) 1 . O diagnóstico é confirmado com:

- 2 critérios clínicos principais mais 1 critério laboratorial;

- 1 critério clínico principal, 1 critério clínico secundário e 1 critério laboratorial.

A RSC compreende diversas doenças com diferentes fenótipos e endótipos, muitos deles, como a RSC com polipose nasal (RSCcPN), caracterizados pela inflamação Tipo $\|^{2}$. A RSCcPN tem prevalência estimada de 4,2\% nos Estados Unidos e de 4,3\% na Europa ${ }^{3,4}$. Geralmente está associada a quadro de obstrução e secreção nasal, além de perda de olfato, de difícil controle. Até $65 \%$ dos pacientes com RSCcPN apresentam asma, e 16\% têm doença respiratória exacerbada pelos anti-inflamatórios não esteroidais (DREA) ${ }^{5,6}$. Os pacientes mais graves recebem cursos repetitivos de corticosteroides sistêmicos ou são dependentes destas medicações, além de serem recorrentemente submetidos a cirurgias para polipectomia e alívio da obstrução nasal. Para estes pacientes as recomendações da European Position Paper on Rhinosinusitis and Nasal Polyps (EPOS) 2020 sugerem o uso de imunobilógicos em pacientes que apresentam três dos seguintes critérios: evidências de inflamação tipo II; necessidade ou contraindicação para o uso de corticosteroides sistêmicos; diminuição significativa da qualidade de vida; perda de olfato significativa; associação com a comorbidade asma ${ }^{1}$.
Três citocinas são fundamentais no desenvolvimento da resposta imune Tipo II: as interleucinas (IL) 4, 5 e 13. O dupilumabe é um anticorpo monoclonal IgG4 totalmente humano, cujo alvo é a unidade alfa do receptor da IL-4. Como esta unidade é comum aos receptores da IL-4 e IL-13, duas citocinas com diversas ações redundantes e que orquestram a inflamação Tipo II, há o bloqueio da sinalização de ambas com a administração do dupilumabe. O desenvolvimento deste imunobiológico representa um avanço no tratamento das doenças atópicas.

Dois estudos clínicos fase 3 , randomizados, duplocegos e controlados com placebo comprovaram a eficácia e a segurança do tratamento de pacientes com RSCcPN com o dupilumabe: LIBERTY NP SINUS-24 e LIBERTY NP SINUS-52. Os estudos incluíram pacientes com RSCcPN grave e não controlada, apesar do tratamento otimizado com corticosteroide nasal, $100 \mu \mathrm{g}$ de furoato de mometasona em cada narina de 12/12 horas. Os pacientes requeriam múltiplos ciclos de corticosteroides sistêmicos e/ou tinham se submetido a cirurgia nasossinusal. Ambos os estudos foram multicêntricos, com 67 centros em diversos países, incluindo a Argentina, Chile e México na América Latina7.

No estudo SINUS-24 os pacientes foram randomizados para receber placebo ou $300 \mathrm{mg}$ de dupilumabe a cada 2 semanas por 24 semanas, com um seguimento de mais 24 semanas após o tratamento. Já no SINUS- 52 os pacientes foram randomizados em três grupos, sendo que: o grupo A recebeu $300 \mathrm{mg}$ de dupilumabe a cada 2 semanas por 52 semanas; o grupo

Tabela 1

Critérios clínico-laboratoriais para o diagnóstico de rinossinusite crônica

\begin{tabular}{lll}
\hline Critério clínico principal & Critério clínico secundário & Critério laboratorial \\
\hline $\begin{array}{l}\text { Bloqueio nasal } \\
\text { (obstrução/congestão nasal) }\end{array}$ & Dor/pressão facial & Endoscopia nasal evidenciando pólipos e/ou \\
& & $\begin{array}{l}\text { secreção nasal do meato médio e/ou edema } \\
\text { com obstrução do meato médio }\end{array}$ \\
$\begin{array}{l}\text { Secreção nasal } \\
\text { (anterior ou posterior) }\end{array}$ & Redução ou perda do olfato & $\begin{array}{l}\text { Tomografia computadorizada com alterações } \\
\text { da mucosa nasal comprometendo o complexo } \\
\end{array}$ \\
& & osteomeatal e/ou os seios paranasais
\end{tabular}


B recebeu $300 \mathrm{mg}$ de dupilumabe a cada 2 semanas por 24 semanas e, depois, a cada 4 semanas até completar as 52 semanas; e o grupo $\mathrm{C}$ recebeu placebo a cada 2 semanas por 52 semanas. Os pacientes foram seguidos por mais 12 semanas após o período de tratamento. A Tabela 2 mostra os desfechos principais e secundários dos dois estudos ${ }^{7}$.

Os estudos SINUS-24 e SINUS-52 incluíram 276 e 448 pacientes, respectivamente. Dentre os 724 pacientes, $59 \%$ tinham asma, e $28 \%$ tinham DREA. Os pacientes tratados com dupilumabe apresentaram melhora significativa, em relação ao placebo, dos escores endoscópico de pólipos nasais e de gravidade da obstrução nasal; melhora já observada entre a quarta e oitava semana de tratamento, mas que foi progressiva até o final do estudo (Figura 1). Também houve melhoras progressivas dos desfechos secundários a partir da segunda semana do estudo? ${ }^{7}$.

A proporção de pacientes que necessitaram cursos de corticosteroide sistêmico e/ou cirugia nasossinusal durante o período de tratamento dos estudos foi estatisticamente menor nos grupos tratados com dupilumabe a cada 2 semanas em relação aos que receberam placebo (Figura 2). Enquanto no estudo
SINUS-24 os efeitos benéficos do anticorpo monoclonal diminuíram após a descontinuação do tratamento, no estudo SINUS-52 houve melhora progressiva dos desfechos do estudo observada nos pacientes que receberam dupilumabe. Observou-se maior eficácia do imunobiológico nos pacientes que receberam as doses a cada duas semanas em relação àqueles que mudaram a posologia para administrações a cada 4 semanas após a semana $24^{7}$. Nos pacientes que tinham asma como comorbidade da RSCcPN, o dupilumabe acarretou a melhora da função pulmonar $\left(\mathrm{VEF}_{1}\right)$ e do controle da asma (ACQ-6), independentemente dos níveis sanguíneos basais de eosinófilos ${ }^{7}$.

Os pacientes que receberam dupilumabe no estudo SINUS-52 apresentaram diminuição sérica da IgE total, periostina, TARC (Thymus and activationregulated chemokine) e eotaxina-3, além de redução da proteína catiônica eosinofílica, IgE total, eotaxina-3 e IL-5 na secreção nasal. Alguns pacientes apresentaram aumento transitório dos eosinófilos sanguíneos.

Conjuntivite foi observada como reação adversa em 2,7\% dos pacientes que receberam o anticorpo monoclonal. Os quadros foram leves e não acarretaram descontinuação da medicação.

\section{Tabela 2}

Desfechos principais e secundários dos dois estudos

\begin{tabular}{|c|c|c|}
\hline Desfecho & SINUS-24 & SINUS-52 \\
\hline Nasal polyp score (NPS)* & + & + \\
\hline Gravidade da obstrução nasal* & + & + \\
\hline Escore tomográfico Lund-Mackay & + & + \\
\hline Perda de olfato & + & + \\
\hline Escore SNOT-22 & + & + \\
\hline Teste de olfato UPSIT & + & + \\
\hline Pico de fluxo nasal & + & + \\
\hline Necessidade de COs ou cirurgia & + & + \\
\hline $\mathrm{VEF}_{1}$ & + & + \\
\hline ACQ-6 & + & + \\
\hline 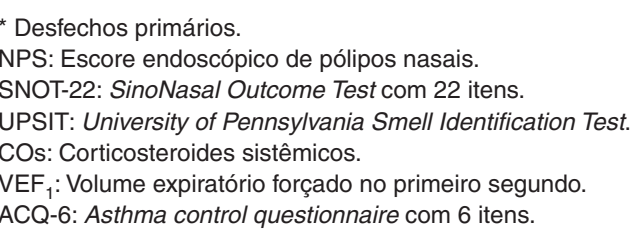 & & \\
\hline
\end{tabular}



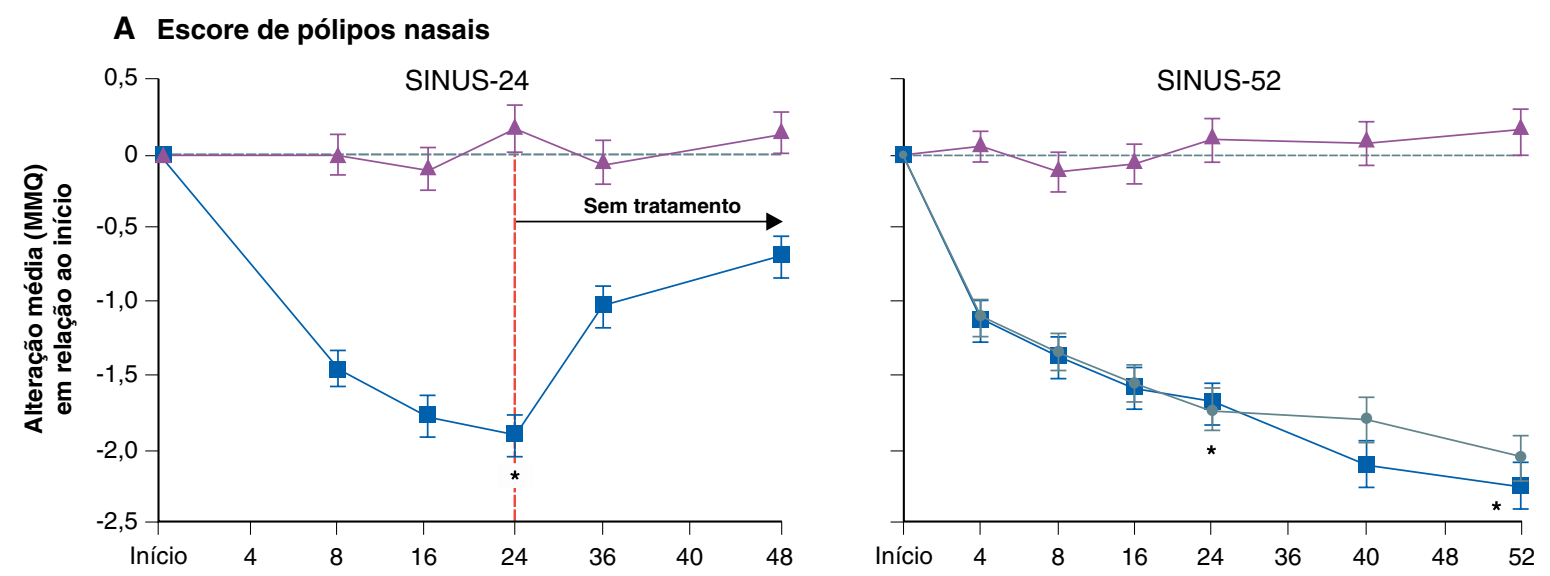

\section{B Congestão ou obstrução nasal}
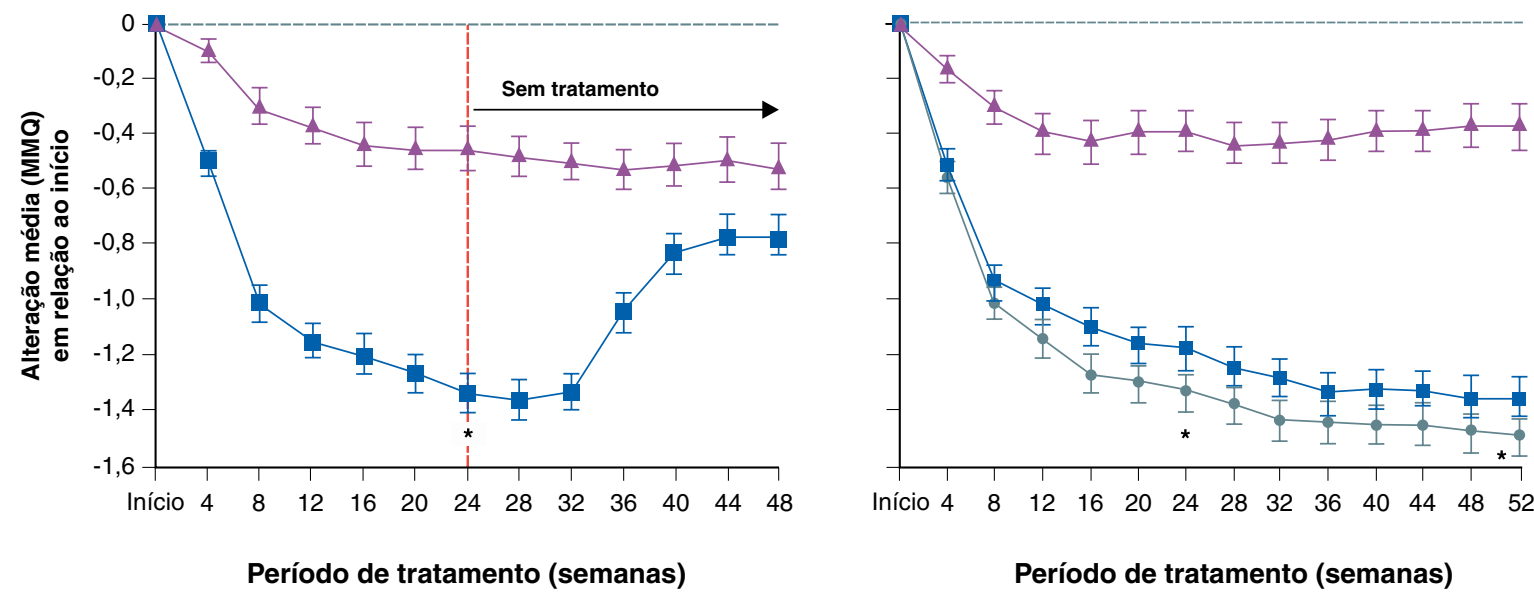

Período de tratamento (semanas)

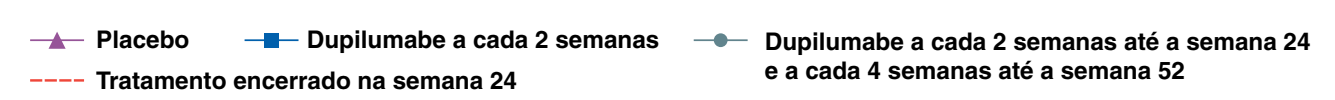

\section{Figura 1}

Alterações ao longo do tempo dos escores endoscópico de pólipos nasais $(A)$ e de gravidade da obstrução nasal (B) nos estudos SINUS-24 e SINUS-52 ${ }^{*} p<0,0001$.

\section{Conclusões}

O dupilumabe é eficaz e seguro no tratamento da RSCcPN. A eficácia é progressiva no primeiro ano de tratamento, e a posologia de $300 \mathrm{mg}$ a cada 2 semanas é superior do que a cada 4 semanas. A interrupção do tratamento com 24 semanas acarreta a perda parcial de seus efeitos benéficos. $\mathrm{O}$ imunobiológico também é eficaz no controle da asma nos pacientes que apresentam esta doença como comorbidade. Alguns pacientes podem apesentar aumento transitório dos eosinófilos sanguíneos, e $2,7 \%$ desenvolveu conjuntivite como reação adversa nos estudos SINUS-24 e SINUS-52.

O dupilumabe é uma excelente opção terapêutica para o tratamento concomitante de múltiplas doenças caracterizadas pela inflamação do Tipo II. 


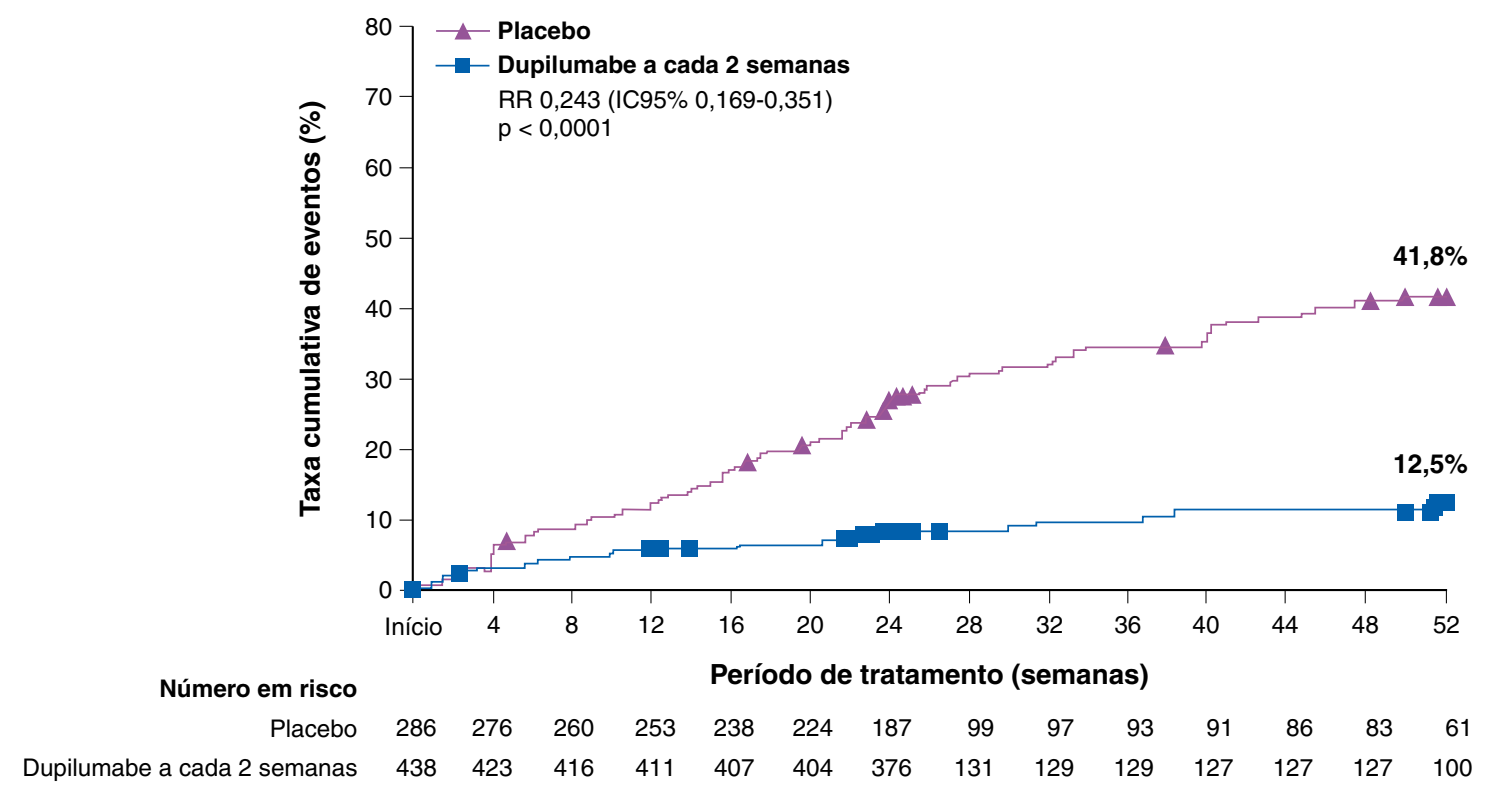

\section{Figura 2}

Tempo até o uso de corticosteroide sistêmico ou cirurgia nasossinusal durante o período de tratamento $\mathrm{RR}=$ razão de risco.

\section{Referências}

1. Fokkens WJ, Lund VJ, Hopkins C, Hellings PW, Kern R, Reitsma S, et al. European Position Paper on Rhinosinusitis and Nasal Polyps 2020. Rhinology. 2020;58(Suppl S29):1-464.

2. Tomassen P, Vandeplas G, Van Zele T, Cardell LO, Arebro J, Olze $\mathrm{H}$, et al. Inflammatory endotypes of chronic rhinosinusitis based on cluster analysis of biomarkers. J Allergy Clin Immunol. 2016;137(5):1449-56.e4.

3. Settipane GA, Chafee FH. Nasal polyps in asthmaand rhinitis. A review of 6,037 patients. J Allergy Clin Immunol. 1977;59(1):17-21.

4. Hedman J, Kaprio J, Poussa T, Nieminen MM. Prevalence of asthma, aspirin intolerance, nasal polyposis and chronic obstructive pulmonary disease in a population-based study. Int $\mathrm{J}$ Epidemiol. 1999;28(4):717-22.

5. Håkansson K, Thomsen SF, Konge L, Mortensen J, Backer V, von Buchwald C. A comparative and descriptive study of asthma in chronic rhinosinusitis with nasal polyps. Am J Rhinol Allergy. 2014;28(5):383-7.
6. Stevens WW, Peters AT, Hirsch AG, Nordberg CM, Schwartz BS, Mercer DG, et al. Clinical Characteristics of Patients with Chronic Rhinosinusitis with Nasal Polyps, Asthma, and AspirinExacerbated Respiratory Disease. J Allergy Clin Immunol Pract. 2017;5(4):1061-70.e3.

7. Bachert C, Han JK, Desrosiers M, Hellings PW, Amin N, Lee SE, et al. Efficacy and safety of dupilumab in patients with severe chronic rhinosinusitis with nasal polyps (LIBERTY NP SINUS-24 and LIBERTY NP SINUS-52): results from two multicentre, randomised, double-blind, placebo-controlled, parallel-group phase 3 trials. Lancet. 2019;394(10209):1638-50.

Não foram declarados conflitos de interesse associados à publicação deste artigo.

Correspondência:

Juliana F. Bianchini

E-mail: jufbianchini@ hotmail.com 